\title{
Myelin oligodendrocyte glycoprotein antibody-associated disorders: clinical spectrum, diagnostic evaluation, and treatment options
}

Yun-Jin Lee, MD, PhD, Sang Ook Nam, MD, PhD, Ara Ko, MD, JuHyun Kong, MD, Shin Yun Byun, MD, PhD

Department of Pediatrics, Pusan National University Children's Hospital, Pusan National University College of Medicine, Yangsan, Korea

Inflammatory or immune-mediated demyelinating central nervous system (CNS) syndromes include a broad spectrum of clinical phenotype and different overlapping diseases. Antibodies against myelin oligodendrocyte glycoprotein (MOG-Ab) have been found in some cases of these demyelinating diseases, particularly in children. MOG-Ab is associated with a wider clinical phenotype not limited to neuromyelitis optica spectrum disorder, with most patients presenting with optic neuritis, acute disseminated encephalomyelitis (ADEM) or ADEM-like encephalitis with brain demyelinating lesions, and/or myelitis. Using specific cell-based assays, MOG-Ab is becoming a potential biomarker of inflammatory demyelinating disorders of the CNS. A humoral immune reaction against MOG was recently found in monophasic diseases and recurrent/multiphasic clinical progression, particularly in pediatric patients. This review summarizes the data regarding MOG-Ab as an impending biological marker for discriminating between these diverse demyelinating CNS diseases and discusses recent developments, clinical applications, and findings regarding the immunopathogenesis of MOG-Ab-associated disorders.

Key words: Myelin oligodendrocyte glycoprotein, Demyelinating diseases, Neuromyelitis optica, Optic neuritis, Acute disseminated encephalomyelitis

\section{Key message}

MOG antibody-associated disorder exhibits different pathophysiological and phenotypic findings than both aquaporin-4 antibody-associated neuromyelitis optica spectrum disorder and typical MS. MOG-antibody is of particular interest in pediatric patients with clinical or radiological non-MS typical findings. MOG-antibody was included in a diagnostic algorithm for children recommending for the first time a standardized use in clinical practice except in cases of typical MS.

\section{Introduction}

Inflammatory or immune-mediated demyelinating central nervous system (CNS) diseases are a heterogeneous group that includes mono- and multiphasic diseases with prognoses ranging from benign to fulminant and a variety of different treatment responses. The most common demyelinating CNS diseases have some chance of misdiagnosis that occurs in up to $10 \%$ of patients. ${ }^{1)}$ Diagnosis is based on a combination of clinical manifestation and radiological and laboratory findings. In 2004, the specific autoantibodies against aquaporin-4 (AQP4), a plentiful water channel on astrocytic endfeet in the CNS, in patients with neuromyelitis optica spectrum disorder (NMOSD) fortified the diagnosis of and research into demyelinating CNS diseases. ${ }^{2}$ However, a subgroup of clinically definite NMOSD patients showed AQP4-seronegative results. ${ }^{3)}$

Myelin oligodendrocyte glycoprotein (MOG) is exclusively expressed in the CNS on the outer surface of the myelin sheath and oligodendrocyte plasma membrane. ${ }^{4}$ MOG was comprehensively studied as a potential target structure in CNS demyelinating diseases. The majority of MOG antibody (MOG-Ab)-seropositive patients have optic neuritis $(\mathrm{ON})$, encephalitis with brain demyelinating lesions, and/or transverse myelitis (TM); the new term "MOG-Ab-associated ON, encephalitis, and myelitis" has been suggested to include these patients with CNS demyelinating syndromes and MOG-Ab positivity. Depending on the clinical assessment, patients can be clinically diagnosed with NMOSD, acute demyelinating encephalomyelitis (ADEM), multiple sclerosis (MS), or isolated ON or TM. ${ }^{5}$ )

In the last few years, the MOG-Ab-associated disorder (MOGAD) spectrum has been rapidly broadening, and as more data regarding their clinical, radiological, and laboratory findings have become available, their immunopathogenesis has been elucidated. This review article discusses the evolving immunopathogenesis, clinical spectrum, diagnostic approach, prognostic research data, and treatment principles for clinical practice.

Corresponding author: Yun-Jin Lee, MD, PhD. Department of Pediatrics, Pusan National University Children's Hospital, 20 Geumo-ro, Mulgeum-eup, Yangsan 50612, Korea 凶Email: jinnyeye@hanmail.net, https://orcid.org/0000-0003-4727-7018

Received: 14 October, 2019, Revised: 16 February, 2020, Accepted: 28 February, 2020

This is an open-access article distributed under the terms of the Creative Commons Attribution Non-Commercial License (http://creativecommons.org/licenses/bync/4.0/) which permits unrestricted non-commercial use, distribution, and reproduction in any medium, provided the original work is properly cited.

Copyright (c) 2020 by The Korean Pediatric Society 


\section{MOG-immunoglobulin G: pathogenic role}

MOG, a glycoprotein of the immunoglobulin superfamily, is a component of the CNS myelin sheath, as are myelin basic protein and proteolipid protein. ${ }^{6}$ ) The precise functions of MOG remain to be clarified but likely include roles in the adhesion of myelin fibers, regulation of oligodendrocyte microtubule stability, and modulation of the interaction between myelin and the immune system by the complement pathway. ${ }^{7)}$

In humans, high-titer MOG-immunoglobulin G (MOG-IgG) levels in serum samples seem to efficiently activate the complement cascade in vitro. ${ }^{8)}$ Furthermore, purified IgG from MOGIgG-positive patients, when incubated with oligodendrocytes in vitro, led to obvious cytoskeletal disorganization, further suggesting functional pathogenicity. ${ }^{9)}$ Initial studies revealed a pathogenic effect of the humoral immune response against MOG (human MOG-Ab); these antibodies were able to induce the death of MOG-expressing cells as well as natural killer cell- mediated cell death, with the extent of cell damage dependent on antibody levels. ${ }^{5)}$ In addition, MOG-Ab belongs to the complement binding IgG1 subtype and has been found to activate the complement cascade, leading to complement-dependent destruction of MOG-expressing cells. ${ }^{8}$ The accumulation of MOG-Ab has been described in CNS antigen-presenting cells with subsequent activation of autoreactive $\mathrm{T}$ cells, follow. ed by the induction of peripheral autoreactive T cells. ${ }^{10)}$ Significantly, MOG-Ab alone did not induce inflammation or tissue destruction; rather, their interdependence with $\mathrm{T}$ cells was required to develop their pathogenic potential. ${ }^{10)}$

Despite MOGAD's ability to overlap with the clinical presentation of AQP4-IgG-associated NMOSD, the mechanisms of the two disease groups are likely different. While the pathological hallmark of AQP4-Ab-positive NMOSD is astrocytic damage with secondary oligodendrocyte loss and demyelination, no evidence of astrocytopathy has been reported in MOG-Ab positivity, which was related to the inflammation and myelin destruction affecting oligodendrocytes without astrocyte injury. ${ }^{11)}$

To our knowledge, several cases to date with obtainable neuropathology have been described in the literature. ${ }^{12-15)}$ Interestingly, most cases discovered MS pattern II lesions with obvious demyelination, marked infiltration of macrophages (containing myelin degradation products), and $\mathrm{T}$ cells, preoligodendrocytes, and relatively preserved axons and astrocytes. The inflammatory hallmark is an infiltrate consisting of T cells as well as a complement, B cell, and $\operatorname{IgG}^{3)}$ suggestive of a humoral pathogenesis. The clinical presentation of MOG-Ab-positive patients with MS pattern II pathology diverges and includes presentation with clinically isolated syndrome, MS, NMOSD, recurrent longitudinally extensive transverse myelitis (LETM), and atypical inflammatory demyelinating CNS syndromes. ${ }^{12-15)}$
Table 1. Clinical and neuroimaging findings indicative of MOG$A b$ versus AQP4-Ab in neuromyelitis optica spectrum disorder

Male sex

Caucasian ethnicity

Single attack or only a few attacks

Bilateral or recurrent optic neuritis sparing the optic chiasm

Longitudinally extensive transverse myelitis involving the lumbar segment and conus medullaris

Good recovery after attacks

AQP4-Ab, aquaporin-4 antibodies; MOG-Ab, myelin oligodendrocyte glycoprotein antibodies.

Adapted from Dos Passos et al. Neurol 2018;9:217. ${ }^{16)}$

\section{Clinical presentation of MOGAD}

Clinical MOG-Ab-positive patients can present with an NMOSD phenotype. Overall, in AQP4-negative patients, MOGAb had a prevalence of $25 \%$ in subsequent studies. ${ }^{3)}$ In contrast to AQP4-Ab-associated disorders with the well-defined phenotype of NMOSD, those with MOGAD have a less well-defined clinical presentation. The foremost findings that distinguish MOG-Ab from AQP4-Ab NMOSD are summarized in Table $1 .{ }^{16)}$

\section{Prevalence}

The prevalence of MOG-Ab seropositivity among patients with NMOSD has been reported by several studies and varies widely depending on the inclusion criteria. Sato el al. ${ }^{17}$ ) found that 7.4\% of all NMOSD patients were seropositive for MOGIgG, while $64.7 \%$ were seropositive for AQP4-IgG. When alterations are made for patients with specific phenotypes and a lack of AQP4-Ab, the proportion of MOG-Ab-positive patients becomes much higher. Two recent studies reported that $40 \%$ of patients with bilateral or recurrent $\mathrm{ON}$ and negative AQP4-Ab were positive for MOG-Ab. ${ }^{17,18)}$ Regarding AQP4$\mathrm{Ab}$-negative LETM, the described prevalence of MOG-Ab was 7.4\%-23.2\%. ${ }^{17,19)}$ Among children, that prevalence appears to be higher: $50 \%$ for patients with definite NMO and $80 \%$ for patients with recurrent $\mathrm{ON}^{20)}$

Particularly, double positivity (i.e., for both AQP4-Ab and MOG-Ab) is usually not identified using cell-based assays, which suggests that each antibody is present in distinct disease processes. Isolated cases of double positivity are extremely rare and usually have significantly higher relapse rates, long-term disability, and magnetic resonance imaging (MRI) lesion burdens, but these characteristics are more compatible with AQP4-Ab-positive NMOSD. ${ }^{21,22)}$

\section{Demographic features}

The proportion of males is generally higher (47\%-62\%) among MOGAD patients than among those with AQP4-Abpositive NMOSD (only 10\%-15\%). ${ }^{1723)}$ However, a recent multicenter European study reported that 74\% of MOG-Abpositive patients were female, resulting in a female-to-male ratio of 2.8:1. ${ }^{24)}$ However, this ratio of female predominance is lower 
than that of up to 9:1 reported in AQP4-Ab patients. ${ }^{25)}$

Regarding age at onset, some studies documented younger age (around the third decade) in the MOG-Ab group versus the AQP4-Ab group (the fourth decade), while others reported no difference. ${ }^{17,23)}$ A few studies presented the age-dependent clinical phenotype, with a predominance of $\mathrm{ON}$ found in adult-onset MOGAD compared to a ADEM-like patterns in children with better recovery. ${ }^{23,26,27)}$ Seizures and encephalitislike symptoms are more frequent in MOGAD than AQP4-Abpositive disorders. ${ }^{28)}$

\section{Clinical phenotypes}

Initially, the MOG-Ab seemed to be associated with a monophasic course and predominantly present in children with an ADEM-like onset. ${ }^{5,29)}$ In subsequent studies, MOG-Ab were present in a subgroup of patients with ADEM, NMOSD, monophasic and relapsing ON, TM, and demyelinating syndromes overlapping with anti-NMDA receptor encephalitis or glycine receptor alpha 1 subunit antibody-positive ON. ${ }^{5,23,29)}$

Two independent groups have evaluated the categories of clinical phenotypes for MOGAD: ON was the major phenotype (41\%-63\%), followed by LETM (29\%-31\%), NMO (6\%$24 \%$ ), and encephalomyelitis (2\%-6\%), while in AQP4-Abpositive patients, the reported phenotypes were $\mathrm{NMO}$ in approximately 60\%, followed by LETM (in about 30\%), and ON (in about 10\%). ${ }^{17,24,30)}$ The first attack of TM or ON can be severe. In the largest series, visual acuity was described to be $<0.1$ at least once in $69 \%$ of patients with ON. ${ }^{24)}$ Among patients with TM, motor symptoms were frequent and included tetraparesis in 28\%, paraparesis in 48\%, and severe weakness in $21 \% .^{24)}$ Brainstem involvement and postrema area syndrome (persistent nausea, vomiting, or hiccups), which usually present in NMOSD (around 40\%), has been reported in 6\%-15\% of MOG-Ab-positive patients as well. ${ }^{17,31)}$ In a large cohort of MOG-Ab-positive patients, ADEM (or an ADEM-like episode) was reported as the initial feature in $18 \%$, primarily affecting children. ${ }^{31)}$ Cortical encephalitis was recently described in MOG-Ab-positive patients but not in AQP4-Ab NMOSD. ${ }^{28,32)}$

A humoral immune response against $\mathrm{MOG}$ is only rarely seen in MS. ${ }^{3)}$ MOG-Ab has been reported up to 5\% of patients diagnosed with atypical MS: severe attacks of myelitis, ON, and/ or severe brainstem syndromes, with failure to several diseasemodifying drugs. ${ }^{33)}$ In this subgroup, frequent relapses and insufficient responses to immune therapy seem common.

\section{MOGAD in pediatric patients}

Several clinical conditions compatible with MOGAD have been described in pediatric patients with MOG-Ab positivity, mainly multiphasic ADEM, ADEM followed by ON, relapsing ON, TM, and AQP4-Ab-negative NMOSD. ${ }^{34)}$ Particularly in relapsing diseases, the differentiation of rare conditions-such as NMOSD, multiphasic ADEM or relapsing $\mathrm{ON}$-has im- portant therapeutic counsel. The first evidence for the role of anti-MOG-IgG as biological markers in children was identified by O'Connor et al. in a subgroup of ADEM. ${ }^{35)}$ Some studies have found that MOG-Ab-positive patients different clinical features according to age. ${ }^{36}$ ) They found a bimodal distribution in 13 pediatric MOG-Ab-positive patients, with encephalopathy being more common in younger patients ( $4-8$ years) and $\mathrm{ON}$ in older patients (13-18 years). ${ }^{36}$ )

Overall, 39\% of MOG-Ab-positive children have a recurrent disease course versus only 5\% with a typical MS disease course. The serial analysis of MOG-Ab-positive patients was recommended at 6-12 months. As there is no gold standard for MOG antibody analysis, particularly for children, the clinical and laboratory findings suggestive of MOGAD retesting is reasonable. As the MOG-Ab titers found in pediatric MS are usually lower and MS diagnostic criteria differ, ${ }^{37)}$ there is no clear association between MOG-Ab and MS, even in pediatric patients.

\section{Disease course}

A preceding infectious episode was noticed in 47\% (e.g., influenza, infectious mononucleosis, following vaccination), with median follow-up times of $12-24$ months. ${ }^{38)}$ The proportion of patients with a single attack is likely reduced with long-term follow-up, in which the proportion of patients with a single attack was only 29\% during a longer median follow-up time (43 months). ${ }^{30)}$ The median time between the first and second attacks was longer in MOG-Ab-positive patients (11.3 years) than in AQP4-Ab-positive (3.2 years) or double seronegative (3.4 years) patients. ${ }^{29}$ ) However, other study revealed the median time between the first and second attacks in MOGAD was 5 months, although the interval could be longer (more than 12 months in 8 patients and up to 492 months in one patient). ${ }^{24)}$

\section{Laboratory evaluation of MOGADs}

\section{MRI findings}

On brain MRI, supratentorial lesions were found in the brainstem of nearly half of patients, while cerebellar lesions were noted in one third. ${ }^{24,27,39)}$ Lesions involving the deep gray matter and lesions adjacent to the fourth ventricle were noted more frequently in AQP4-Ab NMOSD than in the MOGAD. 23) It has become possible to distinguish MOGAD from MS with a specificity of $95 \%$ and a sensitivity of $91 \%$ by different lesion distributions on MRI. Typical MS showed ovoid lesions adjacent to the body of the lateral ventricles, Dawson's fingers (periventricular demyelinating plaques along the axis), and T1 hypointense lesions, whereas fluffy lesions and three or less lesions were typical for MOGAD. ${ }^{40)}$ Lesion distribution in children seems to be age-dependent, with poorly demarcated, widespread lesions in younger children in contrast with a normal result in older children. ${ }^{41)}$ 
On orbital MRI, typical imaging of MOG-Ab-positive ON involve contrast enhancement of the optic nerve, perineural enhancement, and in $80 \%$, more than half of the prechiasmic optic nerve length being affected, sparing the optic chiasm. ${ }^{42)}$ On spinal MRI, while patients with AQP4-Ab usually present cervical (with or without brainstem involvement) and thoracic lesions, MOG-Ab-positive patients can present lesions of the lower cord, including the conus medullaris. ${ }^{17,23)}$ Not all cases of MOG-Ab-positive TM are longitudinally extensive. A small proportion (7\%) of MOG-Ab positivity were described to present with short myelitis occurring after an initial LETM episode or isolated at disease onset. ${ }^{30)}$

\section{Cerebrospinal fluid results}

Cerebrospinal fluid (CSF) reactivity to MOG has only been shown in patients with high serum levels. ${ }^{5,43)}$ Positivity for MOG-Ab in the CSF was found in $71 \%$ of patients who were MOG-Ab-seropositive, with a median CSF MOG-Ab titer of $1: 4$, lower than the serum titer. ${ }^{38)} \operatorname{IgG}$ index, evidence of intrathecal synthesis, were generally absent. ${ }^{38)}$ These findings suggest a peripheral production of MOG-Ab and secondary diffusion in the CNS similar to that in NMOSD. ${ }^{44)}$ Findings of routine CSF analyses were comparable between MOGAD and NMOSD. CSF pleocytosis was detected in 55\%-70\%, with lymphocytic predominance, ${ }^{23,24)}$ and cell counts higher than in typical MS. 24,45) Oligoclonal bands (OCBs) are uncommon (about 10\%). Similarities were found between CSF cytokine profiles in MOGAD and AQP4-Ab-associated NMOSD, with a predominant up-regulation of $\mathrm{T}$ helper 17-related cytokines in the latter, whereas in MS, T helper 1-related cytokines were found. ${ }^{45)}$

\section{Test for MOG antibodies}

Several detection methods have been used to identify MOG$\mathrm{Ab}$. Given the inconsistent results generated by the enzymelinked immunosorbent assay and immunoblot in MS patients, these techniques are not recommended. ${ }^{46)}$ Reliable results have been verified with cell-based assays, the detection of antibodies targeting human MOG using immunofluorescence or fluorescence-activated cell sorting. A recent review analyzed the differences in a summary of 26 studies. ${ }^{3)} \mathrm{A}$ humoral response against MOG was predominantly present in ADEM with an overall sensitivity of $36.4 \%$; it could also be detected in a substantial subgroup of patients with AQP4-negative ON, TM and NMOSD, with overall sensitivity of $26.9 \% .{ }^{47)}$

A cutoff value is important, as low levels of MOG-Ab were also measured in healthy and other neurological controls. Most studies have used a cutoff of $\geq 1: 160 .{ }^{47)}$ A higher prognostic specificity was reported using a higher cutoff titer. The introduction of a cutoff at $>1: 1,280$ MOG-Ab increased the specificity for non-MS disorders (96\% 1:160 vs. 100\% 1:1,280) or a recurrent non-MS courses (75\% 1:160 vs. $86 \% 1: 1,280)$. However, this higher cutoff at $>1: 1,280$ decreased the sensitivity for predicting non-MS disorders (from $47 \%$ to $31 \%$ ) and a recurrent non-MS disease course (from 63\% to 46\%).

\section{Prognosis}

In several studies, MOG-Ab was associated with a favorable outcome. ${ }^{17)}$ MOG-Ab-positive patients less frequently suffer motor disability and have a better extended disability status scale (EDSS) score, compared to AQP4-Ab-positive patients. ${ }^{23}$ Although, MOG-Ab-associated $\mathrm{ON}$ is usually a recurrent disease, visual recovery was good; ${ }^{42)}$ the outcome was better in MOG- than in AQP4-Ab-positive patients correlating with a better preserved retinal fiber layer thickness. ${ }^{42)}$ In patients with $\mathrm{TM}$, the presence of MOG-Ab has also been associated with a better recovery from acute attacks, and really similar frequency of severe attacks at the onset to AQP4-Ab-associated disorders. ${ }^{19)}$ However, not all individuals will recover fully. ${ }^{17)}$ In a large cohort of MOG-Ab-positive patients followed for a median 28 months, $28 \%$ were left with permanent bladder dysfunction; $21 \%$ (among males) with erectile dysfunction; 20\% with bowel dysfunction; $16 \%$ with decreased visual acuity in at least one eye; and $5 \%$ with an EDSS score $\geq 6 .{ }^{31}$

Monophasic and recurrent diseases are associated with MOG$\mathrm{Ab}^{48)}$ Older age, female sex, and MRI findings of atypical MS were risk factors for a recurrent disease course in MOGAD. The informed relapse varied across different studies. Relapse was observed in 36\%-80\% of patients, with an annualized relapse rate of $0.2-0.9$, the highest risk in those with ON or NMOSD phenotypes. ${ }^{24,49}$ These relapse rates seem to be lower than those of AQP4-positive patients. ${ }^{17,23,27)}$ These highly variable data of relapse rates may be due to the different characteristics of the enrolled patients among studies.

\section{Diagnostic recommendations and algorithm for MOGADs}

No clear distinctive phenotype has been identified in patients with MOG-Ab. The red flag signs and symptoms for the diag. nosis of MOGAD are summarized in Table 2. Antibodies against MOG and AQP4 should be tested for in patients with clinical symptoms suggestive of NMOSD, such as bilateral ON, severe brainstem involvement, or LETM; in special patient groups with a high risk of NMOSD; if there is evidence of large cerebral lesions; if MS criteria of dissemination in space are not fulfilled; or if brain MRI findings are normal (Table 2). ${ }^{50,51)}$ As a lack of OCBs is a very rare finding in typical MS, MOG antibody testing should be considered in OCB negative MS patients. In pediatric-onset MS, antibody testing can support the diagnosis of AQP4-negative NMOSD or ADEM followed by relapsing ON or including chronic relapsing inflammatory optic neuropathy. Other red flags indicating MOG-Ab testing include lesions with poorly demarcated margins located in the cerebellar peduncle and a leukodystrophy-like MRI pattern. ${ }^{51)}$ In 2017, Hacohen et al. precisely described the routine diagnostic use of MOG-Ab testing for pediatric patients in clinical practice and proposed a diagnostic algorithm for any episode of CNS demyelination. ${ }^{51)}$ 


\begin{abstract}
Clinically symptoms of NMOSD (bilateral ON, severe brainstem involvement, or LETM) ${ }^{\mathrm{a}}$
If there is evidence of large cerebral lesions

If MS criteria of dissemination in space are not fulfilled

If brain MRI is normal

Typical MS phenotype with negative oligoclonal bands

Pediatric onset of MS

ADEM followed by relapsing ON or with chronic relapsing inflammatory optic neuropathy

Symptoms of CNS demyelinating disease with poorly marginated lesions in cerebellar peduncle and a leukodystrophy-like MRI pattern

MOG-Ab, myelin oligodendrocyte glycoprotein antibodies; NMOSD, neuromyelitis optica spectrum disorder; ON, optic neuritis; LETM, longitudinally extensive transverse myelitis; MS, multiple sclerosis; MRI, magnetic resonance imaging; ADEM, acute disseminated encephalomyelitis; CNS, central nervous system.

a) Testing for antibodies against MOG and aquaporin-4 should be recommended.
\end{abstract}

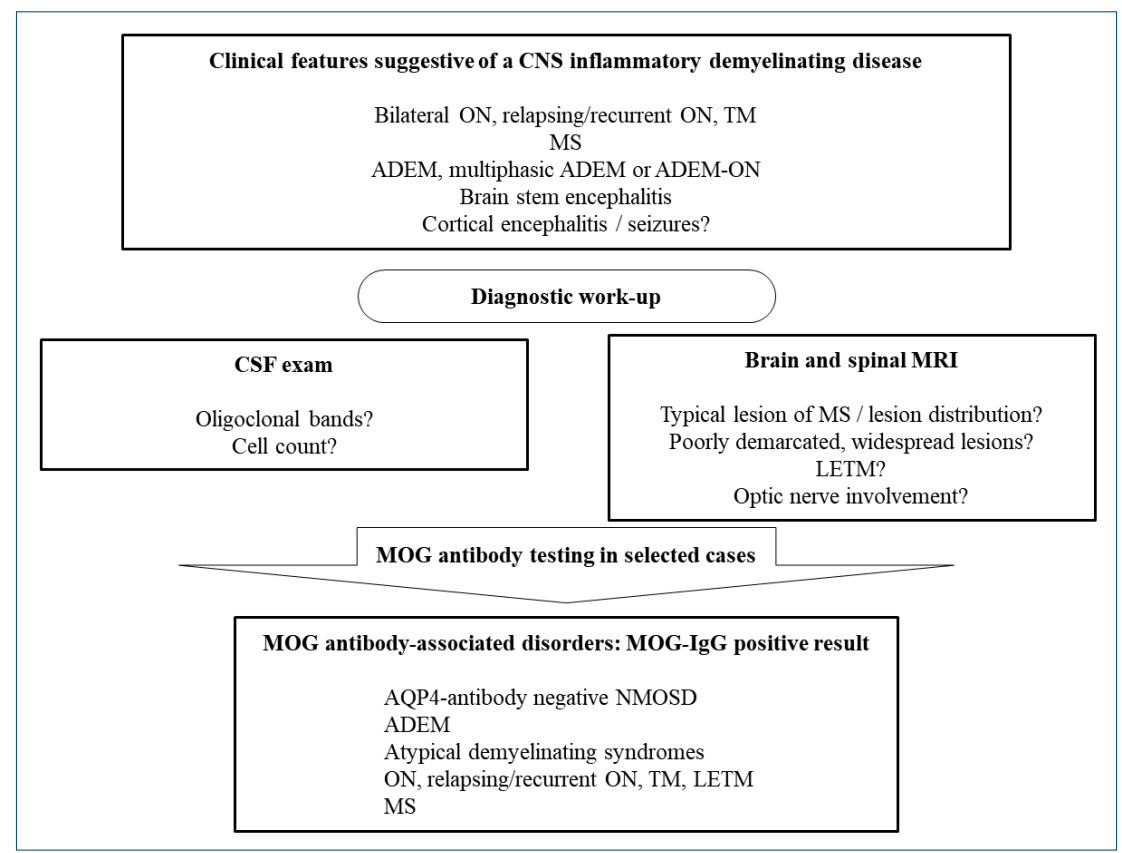

Fig. 1. Diagnostic algorithm and clinical spectrum of MOG antibody-associated disorders. CNS, central nervous system; ON, optic neuritis; TM, transverse myelitis; MS, multiple sclerosis; ADEM, acute disseminated encephalomyelitis; CSF, cerebrospinal fluid; MRI, magnetic resonance imaging; LETM, longitudinally extensive transverse myelitis; MOG, myelin oligodendrocyte glycoprotein; IgG, immunoglobulin G; AQP4, aquaporin-4; NMOSD, neuromyelitis optica spectrum disorder.

In contrast, preceding criteria should include the McDonald criteria with caution for children under 12 years, as the validation of the predictive value is lacking. ${ }^{52)}$

Di Pauli and Berger ${ }^{50)}$ generated a diagnostic algorithm and clinical spectrum of MOGAD in patients with relapsing demyelinating syndromes (Fig. 1). As MS is the most common relapsing demyelinating disorder and reliably diagnosed with brain/spinal MRI and CSF study, brain/spinal MRI and CSF exams are recommended as a first step. In the case of typical MS findings, MS is diagnosed according to the revised McDonald criteria. ${ }^{53)}$ If, in clinically and/or radiologically suggested NMOSD, AQP4-Ab are negative, MOG-Ab should be tested. MOG-Ab testing is also recommended in patients with features of ADEM, particularly those with lesions with poorly demarcated margins located in the cerebellar peduncle or with a leukodystrophy-like MRI pattern. Physicians should be reminded that MOG-Ab-positive patients have distinct clinical features (young, less frequently affected by postrema area syndrome, typically presenting with ADEM initially, less disability during follow-up, and longer time to the first relapse). ${ }^{51)}$

\section{Longitudinal analysis of MOG-antibodies: prognostic and clinical implications}

Predicting the prognosis of MOG-Ab-positive patients considering the possibility of a multiphasic disease course has im. portant suggestions concerning additional initiation of diseasemodifying treatment. The correlation between longitudinal changes in antibody level and clinical progress has been evaluated. ${ }^{29,43)}$ Studies have discovered an association of MOG-Ab titer decrease with a monophasic course compared to stable or increasing titers in patients with multiphasic attacks. ${ }^{27,33)}$ Persistent MOG-Ab has been predominantly found in multiphasic 
ADEM, NMOSD, and ADEM followed by ON. ${ }^{54)}$ One study of adult and pediatric MOG-Ab-positive ADEM patients reinforced the usefulness of serial MOG-Ab testing for predicting relapse, as $88 \%$ of patients with persistent MOG-Ab relapsed during long-term follow-up versus $12 \%$ with transient antibodies. ${ }^{55)}$ These findings indicate that the maintenance of MOG$\mathrm{Ab}$-positivity could predict its clinical progress. On the other hand, Cobo-Calvo et al. ${ }^{27)}$ confirmed the trend toward an association between a relapsing disease and persistent antibodies only in a subgroup; in some groups, no such association was noticed. Similarly, Duignan et al. ${ }^{56}$ ) found persistent MOG-Ab in relapsing and monophasic diseases alike. Long-term studies are needed to evaluate the usefulness of MOG-Ab as a biomarker and follow up the outcomes of demyelinating episodes in the developing CNS.

The conversion to seronegativity of MOG-Ab during immune therapies was a predictive marker for disease-free activity during the subsequent disease course. ${ }^{57)}$ However, more prospective data are needed to confirm the predictive value of serial MOG$\mathrm{Ab}$ testing because the results are in part inconsistent and severe relapsing disease courses have been described in patients with decreasing/disappearing antibody levels.

\section{Treatment}

In an acute attack, similar strategies are used as in other CNS inflammatory or immune-mediated demyelinating diseases (intravenous methylprednisolone, plasma exchange, and intravenous immunoglobulin). A favorable recovery was demonstrated in 70\%-90\% of patients given pulsed intravenous methylprednisolone (dose 20-30 mg/kg/day for children, $500 \mathrm{mg}$ to $1 \mathrm{~g} /$ day for adults over 3-5 days). ${ }^{17,58)}$ Patients were steroid responsive, but $70 \%$ of episodes treated with oral prednisone relapsed, particularly at doses $<10 \mathrm{mg}$ daily or within 2 months of cessation. ${ }^{58)}$ Long-term treatment with corticosteroids reduces the risk of relapse, and cessation has been associated with breakthrough disease. ${ }^{58)}$ Disease worsening or early relapse was observed after corticosteroid tapering or finishing. ${ }^{27,58)}$ Thus, some authors favored long-term oral prednisone treatment over 6 months (starting dose 1-2 mg/kg/day in children, $1 \mathrm{mg} /$ $\mathrm{kg}$ in adults) given alongside other immune-modulating drugs. Use of plasma exchange seems to be associated with improved neurological deficits after corticosteroid failure. ${ }^{33)}$

A challenge in MOGAD is the choice of long-term immunotherapy since the pharmacological options for NMOSD have not been precisely evaluated in patients with MOGAD. Truly, it remains uncertain whether all MOGAD patients will require long-term treatment given the possibility of a monophasic course, the commonly lower relapse rate, and the good recovery after attacks in response to acute treatment. A combination of corticosteroids and other immune-mediated therapies seems favorable. Some studies found a reduction in the annualized relapse rate with different immunotherapies such as azathioprine, rituximab, methotrexate, and mycophenolate, maintenance corticosteroids and rituximab being most effective at preventing disease activity, particularly if treatment is maintained for more than 3 months. ${ }^{27,58)}$ A recent study including children with relapsing MOGAD demonstrated a benefit of intravenous im. munoglobulins on the annualized relapse rate. ${ }^{39)}$ It remains to be determined whether the disease course (i.e., single attack versus further relapses) or higher titers or persistence of MOG-Ab can predict the need for long-term therapy. ${ }^{16)}$

Typical MS drugs such as natalizumab, interferon, and glatiramer acetate revealed no treatment efficacy. ${ }^{24)}$ The failure of treatment efficacy in MS is well-known in NMOSD, suggesting a distinct pathomechanism in antibody-associated disorders.

\section{Conclusion}

Inflammatory demyelinating CNS diseases include a broad spectrum of diverse diseases, among which single diseases might show distinct clinical phenotypes and prognoses. Over the past few years, our knowledge of clinical, imaging, and laboratory data regarding MOGAD has developed. Despite some overlap, MOGAD exhibits different pathophysiological and phenotypic findings than both AQP4-Ab-associated NMOSD and typical MS. A different immunopathogenesis was found in MOG-Abassociated NMOSD, with an oligodendrogliopathy rather than a classical astrocytopathy. This finding is clearly different from AQP4-associated disorders and suggests that other therapeutic strategies might be promising. MOG-Ab is of particular interest in pediatric patients with clinical or radiological non-MS typical findings and reliably predicts a non-MS demyelinating disease course. Indeed, early publications suggested that anti-MOG antibodies argue for a monophasic disease course, although recent data have revealed an association with a risk for recurrence. Since persistent reactivity to MOG indicates a recurrent course, serial testing is recommended. Due to the increasing role of antibody testing, MOG-Ab was included in a diagnostic algorithm for children recommending for the first time a standardized use in clinical practice except in cases of typical MS. Further large prospective studies on the pathophysiology of MOGAD and the role of longitudinal analysis of antibodies are required to enable the development of evidence-based treatment strategies and assess the possible correlation of titers with disease activity.

\section{Conflicts of interest}

No potential conflict of interest relevant to this article was reported.

\section{References}

1. Solomon AJ, Weinshenker BG. Misdiagnosis of multiple sclerosis: frequency, causes, effects, and prevention. Curr Neurol Neurosci Rep 2013; 13:403.

2. Lennon VA, Wingerchuk DM, Kryzer TJ, Pittock SJ, Lucchinetti CF, 
Fujihara K, et al. A serum autoantibody marker of neuromyelitis optica: distinction from multiple sclerosis. Lancet 2004;364:2106-12.

3. Peschl P, Bradl M, Höftberger R, Berger T, Reindl M. Myelin oligodendrocyte glycoprotein: deciphering a target in inflammatory demyelinating diseases. Front Immunol 2017;8:529.

4. Kroepfl JF, Viise LR, Charron AJ, Linington C, Gardinier MV. Investigation of myelin/oligodendrocyte glycoprotein membrane topology. J Neurochem 1996;67:2219-22.

5. Brilot F, Dale RC, Selter RC, Grummel V, Kalluri SR, Aslam M, et al. Antibodies to native myelin oligodendrocyte glycoprotein in children with inflammatory demyelinating central nervous system disease. Ann Neurol 2009;66:833-42.

6. Johns TG, Bernard CC. The structure and function of myelin oligodendrocyte glycoprotein. J Neurochem 1999;72:1-9.

7. Mayer MC, Meinl E. Glycoproteins as targets of autoantibodies in CNS inflammation: MOG and more. Ther Adv Neurol Disord 2012;5:14759.

8. Mader S, Gredler V, Schanda K, Rostasy K, Dujmovic I, Pfaller K, et al. Complement activating antibodies to myelin oligodendrocyte glycoprotein in neuromyelitis optica and related disorders. J Neuroinflammation 2011;8:184.

9. Dale RC, Tantsis EM, Merheb V, Kumaran RY, SinmazN, Pathmanandavel $\mathrm{K}$, et al. Antibodies to MOG have a demyelination phenotype and affect oligodendrocyte cytoskeleton. Neurol Neuroimmunol Neuroinflamm 2014;1:e12.

10. Kinzel S, Lehmann-Horn K, Torke S, Häusler D, Winkler A, Stadelmann $\mathrm{C}$, et al. Myelin-reactive antibodies initiate T cell-mediated CNS autoimmune disease by opsonization of endogenous antigen. Acta Neuropathol 2016;132:43-58.

11. Zamvil SS, Slavin AJ. Does MOG Ig-positive AQP4-seronegative opticospinal inflammatory disease justify a diagnosis of NMO spectrum disorder? Neurol Neuroimmunol Neuroinflamm 2015;2:e62.

12. Wang JJ, Jaunmuktane Z, Mummery C, Brandner S, Leary S, Trip SA. Inflammatory demyelination without astrocyte loss in MOG antibodypositive NMOSD. Neurology 2016;87:229-31.

13. Ikeda T, Yamada K, Ogawa R, Takai Y, Kaneko K, Misu T, et al. The pathological features of MOG antibody-positive cerebral cortical encephalitis as a new spectrum associated with MOG antibodies: a case report. J Neurol Sci 2018;392:113-5.

14. Jarius S, Metz I, König FB, Ruprecht K, Reindl M, Paul F, et al. Screening for MOG-IgG and 27 other anti-glial and anti-neuronal autoantibodies in 'pattern II multiple sclerosis' and brain biopsy findings in a MOG-IgGpositive case. Mult Scler 2016;22:1541-9.

15. Körtvélyessy P, Breu M, Pawlitzki M, Metz I, Heinze HJ, Matzke M, et al. ADEM-like presentation, anti-MOG antibodies, and MS pathology: two case reports. Neurol Neuroimmunol Neuroinflamm 2017;4:e335.

16. Dos Passos GR, Oliveira LM, da Costa BK, Apostolos-Pereira SL, Callegaro D, Fujihara K, et al. MOG-IgG-associated optic neuritis, encephalitis, and myelitis: lessons learned from neuromyelitis optica spectrum disorder. Front Neurol 2018;9:217.

17. Sato DK, Callegaro D, Lana-Peixoto MA, Waters PJ, de Haidar Jorge FM, Takahashi T, et al. Distinction between MOG antibody-positive and AQP4 antibody-positive NMO spectrum disorders. Neurology 2014;82: 474-81.

18. Kim SM, Woodhall MR, Kim JS, Kim SJ, Park KS, Vincent A, et al. Antibodies to MOG in adults with inflammatory demyelinating disease of the CNS. Neurol Neuroimmunol Neuroinflamm 2015;2:e163.

19. Cobo-Calvo Á, Sepúlveda M, Bernard-Valnet R, Ruiz A, Brassat D, Martínez-Yélamos S, et al. Antibodies to myelin oligodendrocyte glycoprotein in aquaporin 4 antibody seronegative longitudinally extensive transverse myelitis: clinical and prognostic implications. Mult Scler 2016; 22:312-9.

20. Rostásy K, Mader S, Hennes EM, Schanda K, Gredler V, Guenther A, et al. Persisting myelin oligodendrocyte glycoprotein antibodies in aquaporin-4 antibody negative pediatric neuromyelitis optica. Mult Scler 2013;19:1052-9.

21. Di Pauli F, Höftberger R, Reindl M, Beer R, Rhomberg P, Schanda K, et al. Fulminant demyelinating encephalomyelitis: insights from antibody studies and neuropathology. Neurol Neuroimmunol Neuroinflamm 2015;2:e175.

22. Yan Y, Li Y, Fu Y, Yang L, Su L, Shi K, et al. Autoantibody to MOG suggests two distinct clinical subtypes of NMOSD. Sci China Life Sci 2016;59:1270-81.

23. Kitley J, Waters P, Woodhall M, Leite MI, Murchison A, George J, et al. Neuromyelitis optica spectrum disorders with aquaporin-4 and myelinoligodendrocyte glycoprotein antibodies: a comparative study. JAMA Neurol 2014;71:276-83.

24. Jarius S, Ruprecht K, Kleiter I, Borisow N, Asgari N, Pitarokoili K, et al. MOG-IgG in NMO and related disorders: a multicenter study of 50 patients. Part 2: epidemiology, clinical presentation, radiological and laboratory features, treatment responses, and long-term outcome. J Neuroinflammation 2016;13:280.

25. Pandit L, Asgari N, Apiwattanakul M, Palace J, Paul F, Leite MI, et al. Demographic and clinical features of neuromyelitis optica: a review. Mult Scler 2015;21:845-53.

26. Chen L, Chen C, Zhong X, Sun X, Zhu H, Li X, et al. Different features between pediatric-onset and adult-onset patients who are seropositive for MOG-IgG: a multicenter study in South China. J Neuroimmunol 2018; 321:83-91.

27. Cobo-Calvo A, Ruiz A, Maillart E, Audoin B, Zephir H, Bourre B, et al. Clinical spectrum and prognostic value of CNS MOG autoimmunity in adults: the MOGADOR study. Neurology 2018;90:e1858-69.

28. Hamid SHM, Whittam D, Saviour M, Alorainy A, Mutch K, Linaker S, et al. Seizures and encephalitis in myelin oligodendrocyte glycoprotein IgG disease vs aquaporin $4 \mathrm{IgG}$ disease. JAMA Neurol 2018;75:65-71.

29. Pröbstel AK, Dornmair K, Bittner R, Sperl P, Jenne D, Magalhaes S, et al. Antibodies to MOG are transient in childhood acute disseminated encephalomyelitis. Neurology 2011;77:580-8.

30. Sepúlveda M, Armangue T, Martinez-Hernandez E, Arrambide G, Sola-Valls N, Sabater L, et al. Clinical spectrum associated with MOG autoimmunity in adults: significance of sharing rodent MOG epitopes. J Neurol 2016;263:1349-60.

31. Jurynczyk M, Messina S, Woodhall MR, Raza N, Everett R, RocaFernandez A, et al. Clinical presentation and prognosis in MOG-antibody disease: a UK study. Brain 2017;140:3128-38.

32. Fukushima N, Suzuki M, Ogawa R, Hayashi K, Takanashi JI, Ohashi T. A case of anti-MOG antibody-positive multiphasic disseminated encephalomyelitis co-occurring with unilateral cerebral cortical encephalitis. Rinsho Shinkeigaku 2017;57:723-8.

33. Spadaro M, Gerdes LA, Krumbholz M, Ertl-Wagner B, Thaler FS, Schuh $\mathrm{E}$, et al. Autoantibodies to MOG in a distinct subgroup of adult multiple sclerosis. Neurol Neuroimmunol Neuroinflamm 2016;3:e257.

34. Baumann M, Hennes EM, Schanda K, Karenfort M, Kornek B, Seidl $\mathrm{R}$, et al. Children with multiphasic disseminated encephalomyelitis and antibodies to the myelin oligodendrocyte glycoprotein (MOG): extending the spectrum of MOG antibody positive diseases. Mult Scler 2016; 22:1821-9.

35. O'Connor KC, McLaughlin KA, De Jager PL, Chitnis T, Bettelli E, Xu C, et al. Self-antigen tetramers discriminate between myelin autoantibodies to native or denatured protein. Nat Med 2007;13:211-7.

36. Fernandez-Carbonell C, Vargas-Lowy D, Musallam A, Healy B, McLaughlin K, Wucherpfennig KW, et al. Clinical and MRI phenotype of children with MOG antibodies. Mult Scler 2016;22:174-84.

37. Ketelslegers IA, Van Pelt DE, Bryde S, Neuteboom RF, CatsmanBerrevoets CE, Hamann D, et al. Anti-MOG antibodies plead against MS diagnosis in an acquired demyelinating syndromes cohort. Mult Scler 2015;21:1513-20.

38. Jarius S, Ruprecht K, Kleiter I, Borisow N, Asgari N, Pitarokoili K, et al. MOG-IgG in NMO and related disorders: a multicenter study of 50 patients. Part 1: frequency, syndrome specificity, influence of disease activity, long-term course, association with AQP4-IgG, and origin. J Neuroinflammation 2016;13:279.

39. Hacohen Y, Wong YY, Lechner C, Jurynczyk M, Wright S, Konuskan B, et al. disease course and treatment responses in children with relapsing 
myelin oligodendrocyte glycoprotein antibody-associated disease. JAMA Neurol 2018;75:478-87.

40. Jurynczyk M, Geraldes R, Probert F, Woodhall MR, Waters P, Tackley G, et al. Distinct brain imaging characteristics of autoantibody-mediated CNS conditions and multiple sclerosis. Brain 2017;140:617-27.

41. Baumann M, Grams A, Djurdjevic T, Wendel EM, Lechner C, Behring B, et al. MRI of the first event in pediatric acquired demyelinating syndromes with antibodies to myelin oligodendrocyte glycoprotein. J Neurol 2018; 265:845-55.

42. Chen JJ, Flanagan EP, Jitprapaikulsan J, López-Chiriboga ASS, Fryer JP, Leavitt JA, et al. Myelin oligodendrocyte glycoprotein antibody-positive optic neuritis: clinical characteristics, radiologic clues, and outcome. Am J Ophthalmol 2018;195:8-15.

43. Di Pauli F, Mader S, Rostasy K, Schanda K, Bajer-Kornek B, Ehling R, et al. Temporal dynamics of anti-MOG antibodies in CNS demyelinating diseases. Clin Immunol 2011;138:247-54

44. Jarius S, Franciotta D, Paul F, Ruprecht K, Bergamaschi R, Rommer PS, et al. Cerebrospinal fluid antibodies to aquaporin-4 in neuromyelitis optica and related disorders: frequency, origin, and diagnostic relevance. J Neuroinflammation 2010;7:52.

45. Kaneko K, Sato DK, Nakashima I, Ogawa R, Akaishi T, Takai Y, et al. CSF cytokine profile in MOG-IgG+ neurological disease is similar to AQP4IgG+ NMOSD but distinct from MS: a cross-sectional study and potential therapeutic implications. J Neurol Neurosurg Psychiatry 2018;89:92736.

46. Berger T, Reindl M. Antibody biomarkers in CNS demyelinating diseases: a long and winding road. Eur J Neurol 2015;22:1162-8.

47. Di Pauli F, Reindl M, Berger T. New clinical implications of anti-myelin oligodendrocyte glycoprotein antibodies in children with CNS demyelinating diseases. Mult Scler Relat Disord 2018;22:35-37.

48. Narayan R, Simpson A, Fritsche K, Salama S, Pardo S, Mealy M, et al. MOG antibody disease: a review of MOG antibody seropositive neuromyelitis optica spectrum disorder. Mult Scler Relat Disord 2018;25:6672.

49. Hamid SHM, Whittam D, Mutch K, Linaker S, Solomon T, Das K, et al. What proportion of AQP4-IgG-negative NMO spectrum disorder patients are MOG-IgG positive?: a cross sectional study of 132 patients. J Neurol 2017;264:2088-94.

50. Di Pauli F, Berger T. Myelin oligodendrocyte glycoprotein antibodyassociated disorders: toward a new spectrum of inflammatory demyeli- nating CNS disorders? Front Immunol 2018;9:2753.

51. Hacohen Y, Mankad K, Chong WK, Barkhof F, Vincent A, Lim M, et al. Diagnostic algorithm for relapsing acquired demyelinating syndromes in children. Neurology 2017;89:269-78.

52. Krupp LB, Tardieu M, Amato MP, Banwell B, Chitnis T, Dale RC, et al. International Pediatric Multiple Sclerosis Study Group criteria for pediatric multiple sclerosis and immune-mediated central nervous system demyelinating disorders: revisions to the 2007 definitions. Mult Scler 2013;19:1261-7.

53. Polman CH, Reingold SC, Banwell B, Clanet M, Cohen JA, Filippi M, et al. Diagnostic criteria for multiple sclerosis: 2010 revisions to the McDonald criteria. Ann Neurol 2011;69:292-302.

54. Hennes EM, Baumann M, Schanda K, Anlar B, Bajer-Kornek B, Blaschek A, et al. Prognostic relevance of MOG antibodies in children with an acquired demyelinating syndrome. Neurology 2017;89:900-8.

55. López-Chiriboga AS, Majed M, Fryer J, Dubey D, McKeon A, Flanagan EP, et al. Association of MOG-IgG serostatus with relapse after acute disseminated encephalomyelitis and proposed diagnostic criteria for MOG-IgG-associated disorders. JAMA Neurol 2018;75:1355-63.

56. Duignan S, Wright S, Rossor T, Cazabon J, Gilmour K, Ciccarelli O, et al. Myelin oligodendrocyte glycoprotein and aquaporin-4 antibodies are highly specific in children with acquired demyelinating syndromes. Dev Med Child Neurol 2018;60:958-62.

57. Hyun JW, Woodhall MR, Kim SH, Jeong IH, Kong B, Kim G, et al. Longitudinal analysis of myelin oligodendrocyte glycoprotein antibodies in CNS inflammatory diseases. J Neurol Neurosurg Psychiatry 2017;88: 811-7.

58. Ramanathan S, Mohammad S, Tantsis E, Nguyen TK, Merheb V, Fung VSC, et al. Clinical course, therapeutic responses and outcomes in relapsing MOG antibody-associated demyelination. J Neurol Neurosurg Psychiatry 2018;89:127-37.

How to cite this article: Lee YJ, Nam SO, Ko A, Kong JH, Byun SY. Myelin oligodendrocyte glycoprotein antibody-associated disorders: clinical spectrum, diagnostic evaluation, and treatment options.Clin Exp Pediatr 2021;64:103-10. https:// doi.org/10.3345/cep.2019.01305 